\title{
Dynamic Valgus Instability of the Knee due to Chronic Medial Collateral Ligament Laxity as a Cause of Recurrent Patella Dislocation
}

\section{Gohil S*, Martineau P and Burman M}

Fiona Stanley and Rockingham Hospitals, Australia

\begin{abstract}
Recurrent patella dislocation can be caused by bony abnormalities, such as patella alta, increased tibial tubercle lateralization, trochlea dysplasia, excessive femoral neck anteversion and external tibial torsion. It can also be caused by soft tissue abnormalities such as a ruptured medial patellofemoral ligament (MPFL) or a weak vastus medialis obliquus. It has been reported in the literature that acute lateral dislocation of the patella can be associated with proximal injury to the medial collateral ligament (MCL).

This article reports the case of a 26-year-old male pro footballer who had recurrent patella dislocation related to
\end{abstract} previous injury to his MPFL and worsened by dynamic valgus knee instability due to chronic MCL insufficiency.

Keywords: Patella dislocation; Medial collateral ligament

\section{Introduction}

Numerous anatomical factors have been identified that predispose to patella dislocation. We present a case of dynamic valgus instability of the knee in a pro footballer secondary to a chronic MCL strain leading to recurrent patella dislocation.

\section{Case report}

A 26-year-old male pro footballer presented to our institution with a history of recurrent patella dislocation. He had a previous attempted medial capsular plication and MPFL repair at another institution 1 years prior. His last dislocation was a month prior and occurred as a valgus force was applied to his knee when a player landed on him as he was tackled.

On clinical examination he was found to have a mild to moderate effusion, good movement with 5 degrees hyperextension to 130 degrees of flexion. He had normal alignment and no abnormalities of his rotational profile. There was a medial scar from previous surgery. He had increase lateral tilt and a hypermobile patella. There was no 'J sign' and no tenderness with a negative patella grind test. There was grade 2 MCL laxity with a spongy endpoint at 30 degrees of flexion and his Q angle increased from 12 to 20 degrees with valgus stress. MCL laxity Grade 1 in extension. Plain radiographs are shown in Figure 1. An MRI scan is shown in Figure 2. Videos 1 and 2 show the hypermobility of his patella and the dynamic increase in his $\mathrm{Q}$ angle with valgus stress respectively.

The patient had not successfully prevented recurrent dislocation with previous surgery, physiotherapy, bracing and a strengthening programme. It was therefore felt that he would benefit from reconstruction of both his MPFL and MCL. Surgery took place in the form of an achilles tendon allograft with bone plug. This was fashioned as a single bone plug with 2 limbs in the shape of a ' $v$ '. A beath pin from the ACL set was used and a $20 \mathrm{~mm}$ by $10 \mathrm{~mm}$ tunnel was created at the level of the medial epicondyle and the bone plug was secured with an interference screw.

The anterior limb was used as the MPFL by drilling 2 transverse 4.5 $\mathrm{mm}$ holes in the top half of the patella. The graft was pulled through laterally from the proximal hole and then medially in the inferior hole and held with interference screws medially in the patella. The posterior limb was looped around a spiked washer in the tibia and sutured onto itself to recreate the MCL, which had no good femoral attachment. This was also reinforced with a suture anchor and a staple (Figure 3).
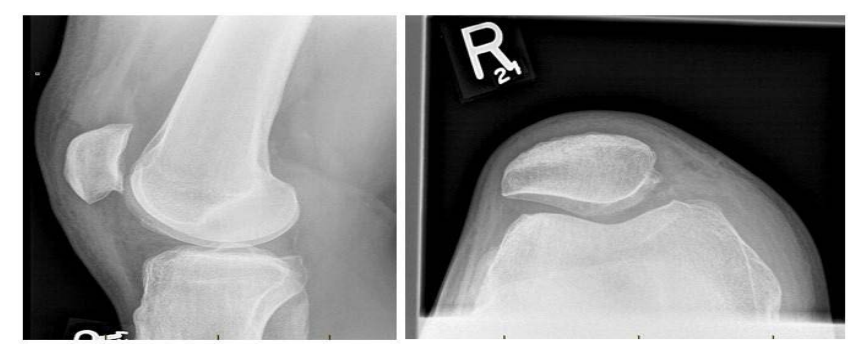

Figure 1: Lateral and skyline view showing degree of patella alta and increased lateral patellar tilt with some early joint degeneration.

He was treated with full weight bearing in a splint in extension for 6 weeks but allowed early range of motion. He then returned back to football at 6 months, managing to compete in the CFL (Canadian Football League)and win the GREY CUP the following season.

\section{Discussion}

Recurrent patellar dislocation can be caused by both bony and soft tissue abnormalities as highlighted in the abstract [1,2]. It has recently been shown that MCL injuries can co-exist following patellar dislocation. It's frequency can be up to $50 \%$ and these are mostly Grade 1 or 2 injuries $[3,4]$. In theory, a valgus force to the knee cause injury both to the femoral attachments of the MPFL and the MCL which are in close proximity to each other.

The medial stabilizers for the patella are both static (bony anatomy) and dynamic (medial retinaculum, MPFL and the vastus medialis obliquus). A chronic deficiency of the medial collateral ligament can be detrimental to the dynamic restraints by causing a relative increase in

*Corresponding author: Satyen Gohil, Consultant Orthopaedic Surgeon, Fiona Stanley and Rockingham Hospitals, Murdoch WA 6150, Australia, Tel: +61 86152 2222; E-mail: satgohil@gmail.com

Received August 27, 2015; Accepted October 10, 2015; Published November 03, 2015

Citation: Gohil S, Martineau P, Burman M (2015) Dynamic Valgus Instability of the Knee due to Chronic Medial Collateral Ligament Laxity as a Cause of Recurrent Patella Dislocation. J Sports Med Doping Stud 6: 168. doi:10.4172/21610673.1000168

Copyright: (C) 2015 Gohil S et al. This is an open-access article distributed unde the terms of the Creative Commons Attribution License, which permits unrestricted use, distribution, and reproduction in any medium, provided the original author and source are credited. 

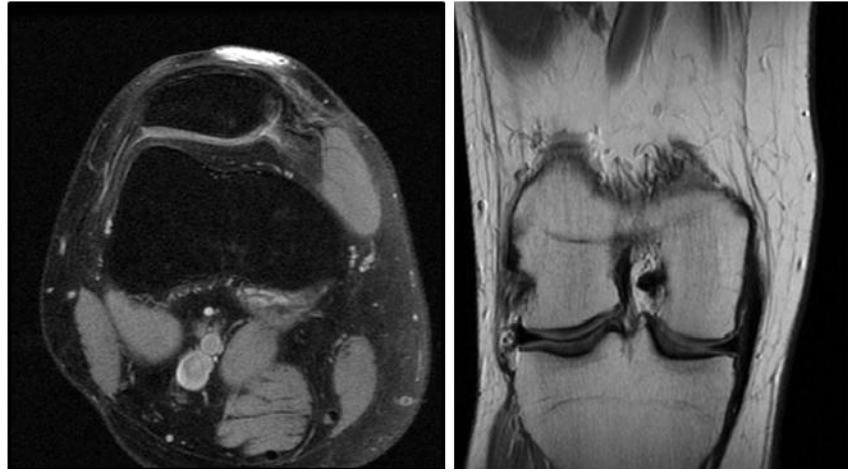

Figure 2: Axial MRI scan on left showing redundant MPFL and medial structures in discontinuity. Coronal MRI scan showing thickening of the femoral attachment of the MCL.

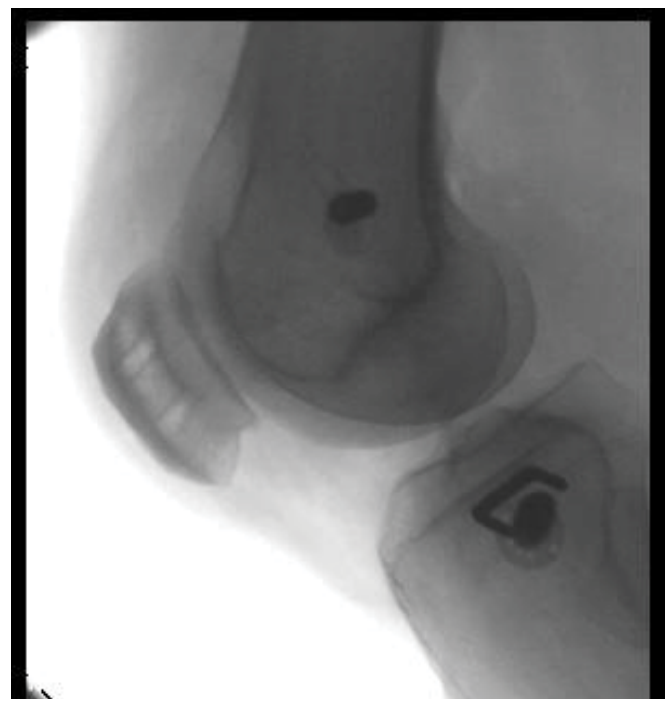

Figure 3: Lateral post-operative radiograph showing the patella drill holes and position of both femoral and tibial fixation.

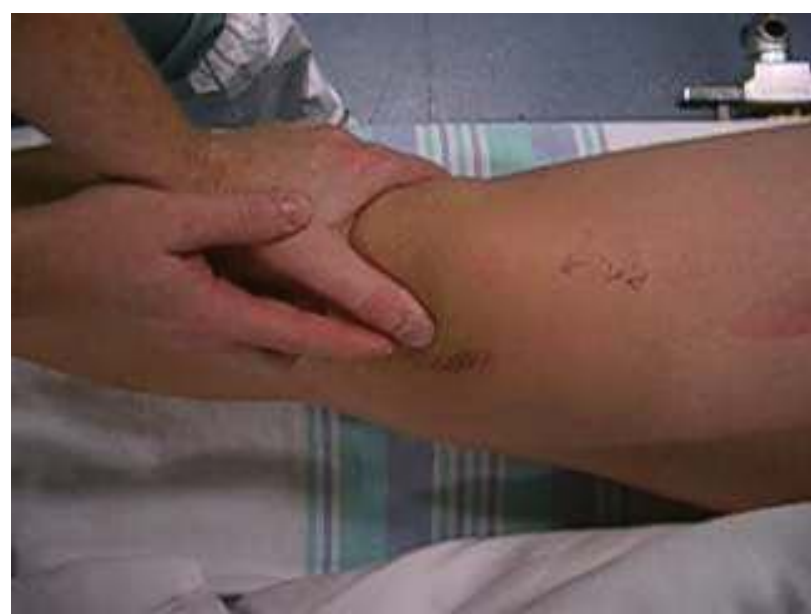

Video 1: Right knee examination showing hypermobility of the patella with increased lateral glide.

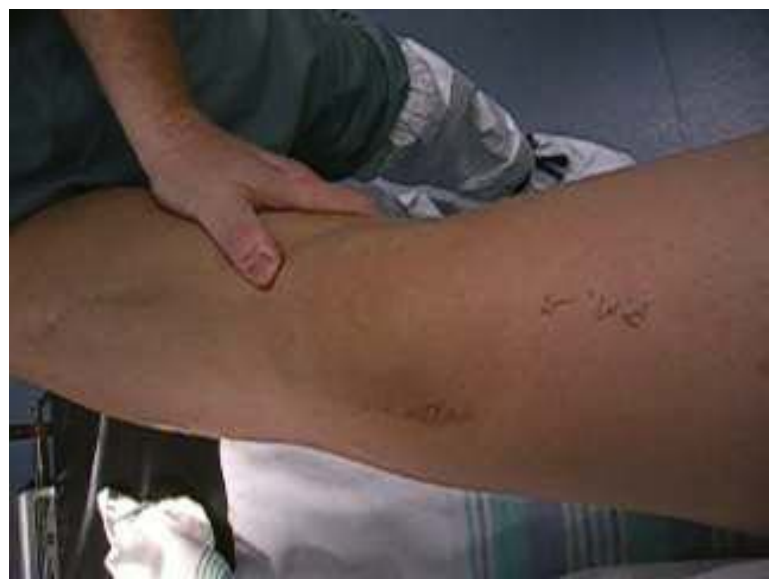

Video 2: Video showing dynamic increase of the $Q$ angle with valgus stress applied to the right knee.

the $\mathrm{Q}$ angle with valgus stress and cause recurrent dislocation.

This case highlights the need to perform a careful clinical examination to assess for concurrent MCL injury following patellar dislocation. MCL injury should also be considered if despite adequate therapy and previous surgery a patient has recurrent patellar dislocations. If both injuries are present and there is residual valgus laxity after 4 to 6 weeks of bracing, it is likely that surgery will be required to address both these problems.

Conflict of interest statement: There were no conflicts of interest related to this case report.

Ethics standard statement: The patient gave verbal consent to the publication of the case study.

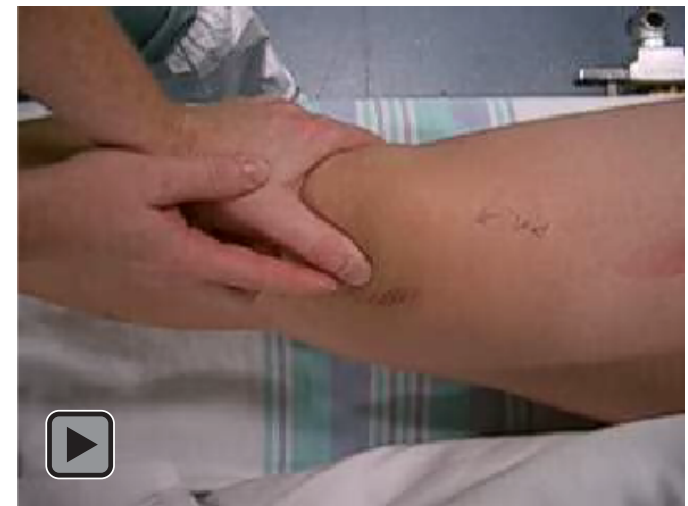




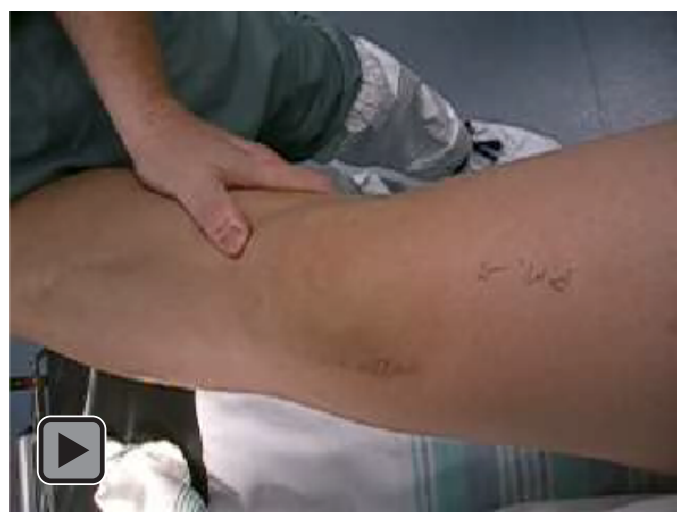

\section{Reference}

1. Smith TO, Song F, Donell ST, Hing CB (2011) Operative versus non-operative management of patellar dislocation. A meta-analysis. Knee Surg Sports Traumatol Arthrosc 19: 988-998.

2. Grelsamer RP (2000) Current concepts review. Patellar malalignment. J Bone Joint Surg Am 82:1639-1650.

3. Quinlan J F, Farrelly C, Kelly G, Eustace S (2010) Co-existent medial collatera ligament injury seen following transient patellar dislocation: observations at magnetic resonance imaging. Br J Sports Med 44: 411-414.

4. Hermans K, Claes S, Bellemans J (2013) Valgus instability as a cause for recurrent lateral patellar dislocation: a new mechanism for patellofemoral instability? Acta Orthop Belg 79: 495-501. 